\title{
Áreas de atuação de pesquisadores como input para caracterizar a atuação institucional
}

\author{
Rosangela Galdino \\ Mestre; Universidade Federal de São Carlos, São Paulo, Brasil; \\ rosangelagaldino@yahoo.com.br \\ Roniberto Morato do Amaral \\ Doutor; Universidade Federal de São Carlos, São Paulo, Brasil; \\ roniberto@ nit.ufscar.br
}

\begin{abstract}
Resumo: A avaliação da atividade científica envolve estudos bibliométricos, que utilizam como fontes de informação bases de dados como, por exemplo, a Web of Science, a Scopus e a Plataforma Lattes. Esta pesquisa visou contribuir para a caracterização da atuação das instituições de ciência, tecnologia e inovação através dos estudos bibliométricos; mais especificamente, seu objetivo geral foi caracterizar o perfil de atuação de uma instituição de ciência, tecnologia e inovação, utilizando as informações sobre as áreas de atuação dos seus docentes-pesquisadores presentes nos seus respectivos currículos Lattes. O método utilizado foi o estudo de caso e a unidade-caso foi o Instituto Federal de Educação, Ciência e Tecnologia de São Paulo (IFSP). A bibliometria foi utilizada como técnica de análise de informações e a Plataforma Lattes foi utilizada como fonte de coleta de dados. A amostra analisada compreendeu 1997 currículos Lattes de docentes-pesquisadores ativos no período de 2010 a 2018. Constatou-se o caráter multidisciplinar da atuação da instituição, bem como a proximidade de atuação entre os seus campi, e traçou-se o perfil disciplinar e multidisciplinar dos docentes. Conclui-se que os resultados alcançados podem contribuir para os estudos bibliométricos de instituições de ciência, tecnologia e inovação, ao sistematizar, através da análise das áreas de atuação dos pesquisadores, a identificação e a visualização de seu perfil de atuação, bem como a identificação de áreas de interesse potencialmente maior para colaboração entre docentes.
\end{abstract}

Palavras-chave: Área de atuação. Currículo Lattes. Instituto Federal de Educação Ciência e Tecnologia de São Paulo. Bibliometria.

\section{Introdução}

No Brasil, a tríade ensino, pesquisa e extensão é intrínseca tanto às universidades quanto aos Institutos Federais de Educação, Ciência e Tecnologia 
(IFs). Nos IFs, a prioridade é a pesquisa aplicada com foco nas esferas econômica, cultural e social (BENTIN, 2017). Assim, a pesquisa é uma das dimensões em que tanto as universidades quanto os IFs atuam, podendo seus resultados serem visualizados, por exemplo, nos estudos bibliométricos, a publicação científica é utilizada como input para a avaliação da atividade de pesquisa (BORDONS; ZULUETA, 1999; ROSTAING, 1996).

Apesar dos avanços alcançados com o uso dos estudos bibliométricos na avaliação da atividade científica, faz-se necessário ampliar tais investigações para compreender a atuação das instituições de ciência, tecnologia e inovação (ICTs) brasileiras. É importante ressaltar que a produção científica é um resultado tangível da atuação de pesquisadores, de seus grupos de pesquisa e das instituições. No entanto, há outros elementos que podem ser utilizados para a avaliação da atividade científica visando ampliar a sua compreensão e o reconhecimento do seu impacto social e econômico na sociedade (SALA, 1991; SOARES, 2018).

Nesse contexto, Torres (2018) destacou as características de institutos públicos de pesquisa e os inputs necessários para a produção científica, utilizando os currículos Lattes (CVLattes) dos seus próprios pesquisadores. Estudos bibliométricos desse tipo podem ser estendidos aos IFs que são ICTs jovens, com uma nova institucionalidade (BENTIN, 2017), ainda em processo de definição e com missão e atuação próprias, que distinguem essas instituições de outras instituições acadêmicas (DOMINGOS SOBRINHO, 2007). Desse modo, as áreas de atuação informadas pelos pesquisadores em seus CVLattes na Plataforma Lattes podem ser utilizadas como input informacional sobre a atuação institucional pelas instituições que desejam conhecer o seu perfil de atuação. Assim, esta pesquisa teve como objetivos principais caracterizar o perfil de atuação de uma ICT, com base na análise das informações sobre as áreas de atuação dos seus pesquisadores, e também, de modo geral, contribuir para os avanços dos estudos bibliométricos e para a maximização do sucesso do projeto institucional de ICTs, através do uso da Plataforma Lattes como fonte de informações. 
Os resultados aqui alcançados podem auxiliar as ICTs a: 1) identificar em que áreas do conhecimento são mais atuantes; 2) descobrir quais áreas podem auxiliá-las a alavancar com maior vigor as iniciativas de pesquisa institucional; 3) visualizar possibilidades de parcerias inter- e intrainstitucionais, com base no conhecimento das áreas e suas relações; 4) facilitar o acesso aos pesquisadores e suas competências, gerando inputs para a identificação de possíveis oportunidades de colaboração entre pesquisadores da instituição. Este processo poderia auxiliar a ampliar e a qualificar a atuação da instituição, além de significar economia de recursos tanto para as ICTs quanto para as instituições de fomento à pesquisa (MAIA; CAREGNATO, 2008).

\section{Referencial teórico}

Os IFs tiveram sua origem nas Escolas de Aprendizes Artífices, instituídas pelo presidente Nilo Peçanha através do decreto $n^{\circ} 7.566$ de 23 de setembro de 1909 (BRASIL, 1909). Ao longo do tempo, houve muitas alterações em sua denominação e dentre os nomes já recebidos estão Liceus Industriais, Escolas Industriais e Técnicas, Escolas Técnicas Federais e Centros Federais de Educação Tecnológica (CEFETs). Finalmente, em dezembro de 2008 foi publicada a Lei $\mathrm{n}^{\circ}$ 11.892, que instituiu a Rede Federal de Educação Profissional, Científica e Tecnológica e criou os IFs.

Os IFs são, no âmbito de sua atuação, “[...] instituições de educação superior, básica e profissional, pluricurriculares e multicampi, especializados na oferta de educação profissional e tecnológica nas diferentes modalidades de ensino [...]" (BRASIL, 2008, p. 1). Vê-se, por essa descrição da finalidade dos IFs, que a formação superior também constitui objeto de atuação dos institutos. Nesse sentido, os IFs se assemelham às universidades.

Assim como nas universidades, a tríade ensino, pesquisa e extensão são intrínsecas aos IFs. $\mathrm{O}$ ato de pesquisar nos IFs está ancorado a princípios regulamentados pelo Ministério da Educação (BRASIL, 2010). No entanto, mesmo sendo tarefa dos IFs realizar atividades de pesquisa, a cultura profissional que os IFs herdaram das instituições que os precederam dificulta a 
inserção da pesquisa entre os focos da atuação do docente, uma vez que esse docente precisa atuar para além do ensino, como historicamente vinha fazendo, e desempenhar uma nova função: a de produtor e difusor do conhecimento científico adequado às necessidades e aos desafios impostos à sociedade atual (FARTES, 2014).

Considerada essa situação, torna-se ainda mais relevante que as atividades de pesquisa dos IFs sejam apoiadas por estudos bibliométricos, que considerem as peculiaridades tanto da instituição quanto dos seus docentespesquisadores. Além disso, também é relevante que os IFs ponderem sobre os desafios que "o fazer ciência" enfrenta no Brasil - escassez de recursos, prazos reduzidos, problemas complexos, etc. (SOARES, 2018). Neste contexto torna-se necessário que as instituições repensem a sua atuação e considerem, entre outras alternativas, o uso racional de recursos por meio da adoção de iniciativas, tais como a colaboração científica (VANZ; STUMPF, 2010).

A colaboração científica possibilita a resolução de problemas complexos de pesquisa, a realização de pesquisa multidisciplinar (e em muitos casos até trans- e interdisciplinar) e o aumento da experiência dos pesquisadores (VANZ; STUMPF, 2010), além de gerar economia de tempo e de recursos financeiros e materiais. Por isso, ela é incentivada pelas agências de financiamento às pesquisas (MAIA; CAREGNATO, 2008).

Resumidamente, a colaboração científica envolve dois ou mais pesquisadores trabalhando juntos com metas e esforços comuns, compartilhando recursos intelectuais, econômicos e físicos e buscando a produção de novos conhecimentos científicos (KATZ; MARTIN, 1997; VANZ; STUMPF, 2010). Quando há o envolvimento apenas de agrupamento de módulos disciplinares, essa colaboração é denominada de multidisciplinar e “[...] só evoca uma simples justaposição, num trabalho determinado, dos recursos de várias disciplinas, sem implicar necessariamente um trabalho de equipe e coordenado [...]" (JAPIASSU, 1976, p. 72). A colaboração é denominada de interdisciplinar quando "[...] a cooperação entre várias disciplinas ou setores heterogêneos na mesma ciência leva a interações reais, a uma certa reciprocidade de trocas resultantes / em enriquecimento mútuo." (PIAGET, 1972, 136-137). Por fim, a 
colaboração é transdisciplinar quando possui “[...] níveis e objetivos múltiplos, coordenando todas as disciplinas e interdisciplinas, [...] tendo em vista uma finalidade comum dos sistemas." (JANTSCH, 1972, p. 75).

Utilizando as informações presentes no CVLattes, Lança, Amaral e Gracioso (2018) investigaram a interdisciplinaridade dos programas de pósgraduação da área de conhecimento da Ciência da Informação no Brasil, analisando e relacionando informações sobre áreas de atuação dos coautores brasileiros que publicam em Ciência da Informação. Os autores concluíram que os pesquisadores dos programas de pós têm perfil multidisciplinar e que diferentes áreas do conhecimento recebem e fornecem contribuições à Ciência da Informação (LANÇA; AMARAL; GRACIOSO, 2018).

O CVLattes pode ser usado como fonte de informações para estudos bibliométricos por conter uma grande riqueza de informações dos pesquisadores do Brasil. Na literatura da área, a Plataforma Lattes é fonte de informações para analisar dados científicos em diversas publicações (FRANCO; FARIAS, 2019; GALDINO; GARCIA; AMARAL, 2019; HADDAD; MENA-CHALCO; SIDONE, 2017; LANÇA; AMARAL; GRACIOSO, 2018; TORRES, 2018).

Assim, apesar de algumas limitações (como a atualização depender do pesquisador, por exemplo, influenciando na confiabilidade dos dados), a Plataforma Lattes é um exemplo de boa prática para os demais países e o CVLattes é um dos melhores bancos de dados sobre pesquisadores que existe na atualidade (LANE, 2010). É por esses motivos que a Plataforma Lattes pode ser utilizada, entre outras funções, para avaliar grupos ou instituições; para mensurar a produção de pesquisadores ou a produção de uma área por meio de estudos bibliométricos; para investigar e compreender grupos de pesquisa, utilizando-se as grandes áreas, áreas, subáreas do conhecimento ou até mesmo todo o banco de dados do CVLattes (BRITO; QUONIAM; MENA-CHALCO, 2016; MENA-CHALCO; DIGIAMPIETRI; CESAR JUNIOR, 2012).

É importante salientar que, durante o preenchimento do CVLattes, o pesquisador pode escolher suas áreas de atuação, compostas pelas grandes áreas do conhecimento, e respectivas áreas, subáreas e especialidades, de acordo com a classificação realizada pela Coordenação de Aperfeiçoamento de Pessoal de 
Nível Superior (Capes) (SIDONE; HADDAD; MENA-CHALCO, 2016). Um estudo bibliométrico baseado nessas informações poderia apontar conjuntos de pesquisadores pertencentes ao mesmo campo de atuação, o que delimitaria as áreas do conhecimento predominantes na instituição, ou seja, o perfil da instituição no que tange à sua atuação. Além disso, se tais conjuntos pertencerem a uma instituição que realiza pesquisa científica e forem criados com base nas subáreas ou especialidades indicadas no CVLattes, a análise desses conjuntos poderia revelar importantes oportunidades de colaboração científica para a instituição.

\section{Metodologia}

A pesquisa classifica-se como exploratória quanto aos seus objetivos, aplicada quanto a seus fins (GIL, 2010) e adota abordagem quantitativa. Além disso, classifica-se como estudo de caso, sendo o Instituto Federal de Educação, Ciência e Tecnologia de São Paulo (IFSP) selecionado como unidade-caso. A escolha da instituição se deve tanto a critérios de conveniência e proximidade geográfica, uma vez que um dos autores possui vínculo com a instituição, quanto à própria importância do IFSP, haja vista seu grande potencial de contribuição para o desenvolvimento da sociedade brasileira.

O desenvolvimento da pesquisa iniciou-se com a identificação dos docentes ativos na instituição, que foi realizada através de levantamento junto à Coordenadoria de Admissão de Pessoal (CAP - Reitoria) do IFSP. Após a organização dos nomes dos docentes, passou-se à etapa de obtenção do código de identificação único (IDs Lattes) dos CVLattes de cada um, realizada manualmente entre os dias 18/09/2018 e 29/11/2018 através de consulta no site da Plataforma Lattes (http://lattes.cnpq.br/).

De posse dos IDs Lattes, para a coleta dos dados dos CVLattes utilizouse o Synclattes, uma ferramenta criada para extrair, tratar e sincronizar os metadados do CVLattes (MATIAS, 2015). A coleta de dados foi limitada ao período de 2010 a 2018, uma vez que foi a partir de 2010 que o IFSP realmente consolidou-se com a criação efetiva da maioria dos campi existentes. Dos 2214 
docentes ativos no IFSP, o resultado da coleta foi de um total de 1997 CVLattes com áreas de atuação definidas. Ainda segundo a coleta, 217 docentes não indicaram nenhuma área de atuação.

Posteriormente, para a organização e tratamento das informações obtidas pelo Synclattes, elas foram importadas para o VantagePoint, uma ferramenta para mineração de textos que permite a limpeza, análise e visualização de grandes quantidades de informações (PORTER; PALOP, 2012). Com o apoio desse software, foi possível gerar uma série de listas, matrizes e mapas necessários à caracterização do perfil de atuação da unidade-caso e, além disso, foi possível através do Microsoft Excel representar visualmente um conjunto de indicadores sobre a área de atuação dos docentes do IFSP. Assim, a bibliometria foi utilizada como técnica de análise de informações em conjunto com o software VantagePoint.

Para tanto, foram realizados dois tipos de análises: uma que considerou apenas a primeira indicação da área de atuação dos docentes ativos, que pode ser considerada como indicador de disciplinaridade, e outra que considerou todas as indicações das áreas de atuação dos docentes separados por campus, que podem ser consideradas como um indicador da multidisciplinaridade.

Os indicadores utilizados nos mapas de correlação cruzada (área e subárea do conhecimento) foram criados utilizando-se todas as áreas de atuação indicadas pelos docentes e seus campi de atuação. De acordo com o VantagePoint (2015, p. 174), “[...] um mapa de correlação cruzada mostra relacionamentos entre itens em uma lista com base nos valores em outra lista [...]". Assim, os mapas de correlação cruzada dos campi, incorporando as áreas e as subáreas indicadas pelos docentes em seus CVLattes, demonstram os grupos de docentes que selecionaram as mesmas áreas e subáreas por campus. Com esse indicador é possível verificar se os campi possuem áreas e subáreas comuns entre si. É importante ressaltar que cada docente pode indicar até seis áreas de atuação e que cada um está relacionado a apenas um campus (à unidade em que efetivamente trabalha). Assim, as correlações ocorrem do cruzamento do campus de cada docente com as áreas e subáreas que indicaram em seu CVLattes. 


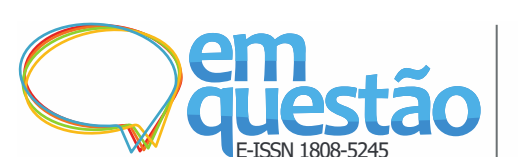

Áreas de atuação de pesquisadores como input para

caracterizar a atuação institucional

Rosangela Galdino, Roniberto Morato do Amaral

\section{Resultados e discussão}

Com os dados dos 1997 CVLattes dos docentes, foi possível extrair as áreas de atuação de cada um deles. O preenchimento da área de atuação não é obrigatório no CVLattes e deve ser realizado pelo próprio docente, que opta por preencher apenas o primeiro nível (grande área), ou os dois primeiros (grande área + área), os três primeiros (grande área + área + subárea) ou todos os quatro níveis da área de atuação (grande área + área + subárea + especialidade).

Esse preenchimento pode acontecer até seis vezes num mesmo currículo. Em outras palavras, cada docente pode preencher até seis áreas de atuação diferentes (no caso do preenchimento de mais de uma, o CVLattes solicita que a primeira opção seja a mais importante para o pesquisador). O Gráfico 1 mostra o percentual da "primeira indicação" dos docentes para as grandes áreas do conhecimento de acordo com a Tabela de Áreas do Conhecimento do Conselho Nacional de Desenvolvimento Científico e Tecnológico (CNPq).

Gráfico 1 - Primeira indicação - grandes áreas

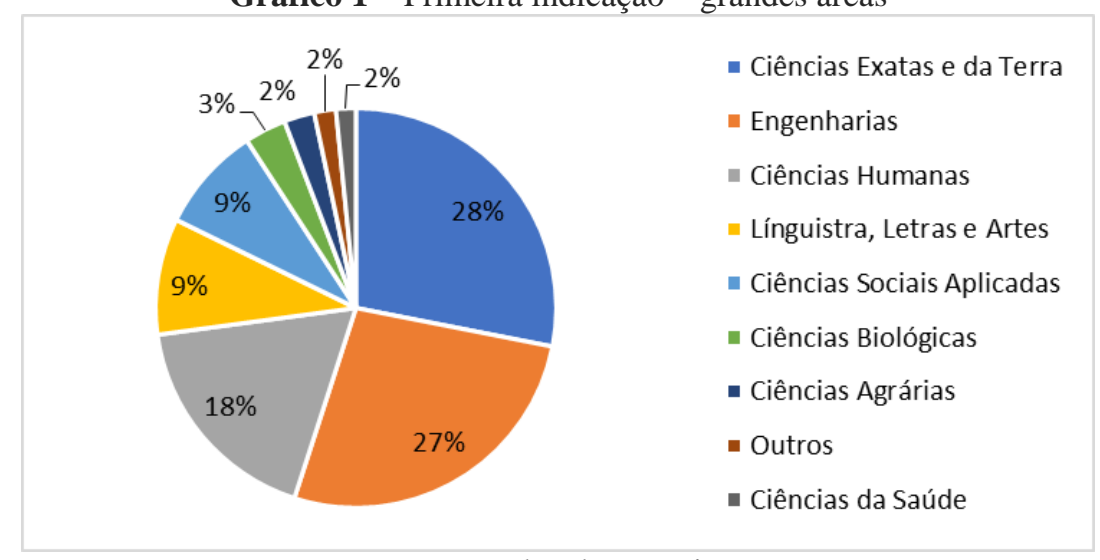

Fonte: Dados da pesquisa.

As grandes áreas Ciências Exatas e da Terra, Engenharias e Ciências Humanas somam juntas $73 \%$ das primeiras indicações do instituto. Isso se justifica pelo fato de a maior quantidade de cursos que o IFSP oferece estar inserida nessas grandes áreas. Abaixo dessas, mas ainda com presença relevante, estão as grandes áreas Ciências Sociais Aplicadas e Linguística, Letras e Artes, que juntas respondem por $18 \%$ de indicações pelos docentes. Além disso, considerando-se que a primeira indicação de área de atuação é a prioritária para 
os pesquisadores, de acordo com os estudos realizados por Torres (2018), as primeiras indicações das grandes áreas também podem ser um indicativo das áreas do conhecimento em que a força de trabalho dos docentes do IFSP mais se concentra, ou seja, Ciências Exatas e da Terra, Engenharias e Ciências

\section{Humanas.}

Ao ser representada como no Gráfico 1, de maneira percentual e com base nas primeiras indicações, a distribuição das grandes áreas pode ser vista como uma primeira representação do peso das diferentes "famílias" de conteúdos disciplinares presentes no IFSP, ou seja, de um “[...] conjunto sistemático e organizado de conhecimentos que apresentam características próprias nos planos do ensino, da formação, dos métodos e das matérias [...]" (JAPIASSU, 1976, p. 72). Sendo a pesquisa de cunho disciplinar a mais comumente encontrada, é de se esperar que o IFSP tenha sua produção científica distribuída de forma aproximada à distribuição de percentuais do gráfico acima.

O Gráfico 2 (Todas as indicações - grandes áreas) mostra o percentual de todas as indicações dos docentes para as grandes áreas, e não apenas a primeira indicação, como no Gráfico 1. Nota-se que não há grandes alterações percentuais com relação ao gráfico anterior, o que indica que há pouca variação da grande área quando um docente indica diferentes áreas de atuação. Em outras palavras, mesmo quando o docente acredita ter duas ou mais áreas de atuação, sua escolha da grande área tende a permanecer a mesma.

Gráfico 2 - Todas as indicações - grandes áreas

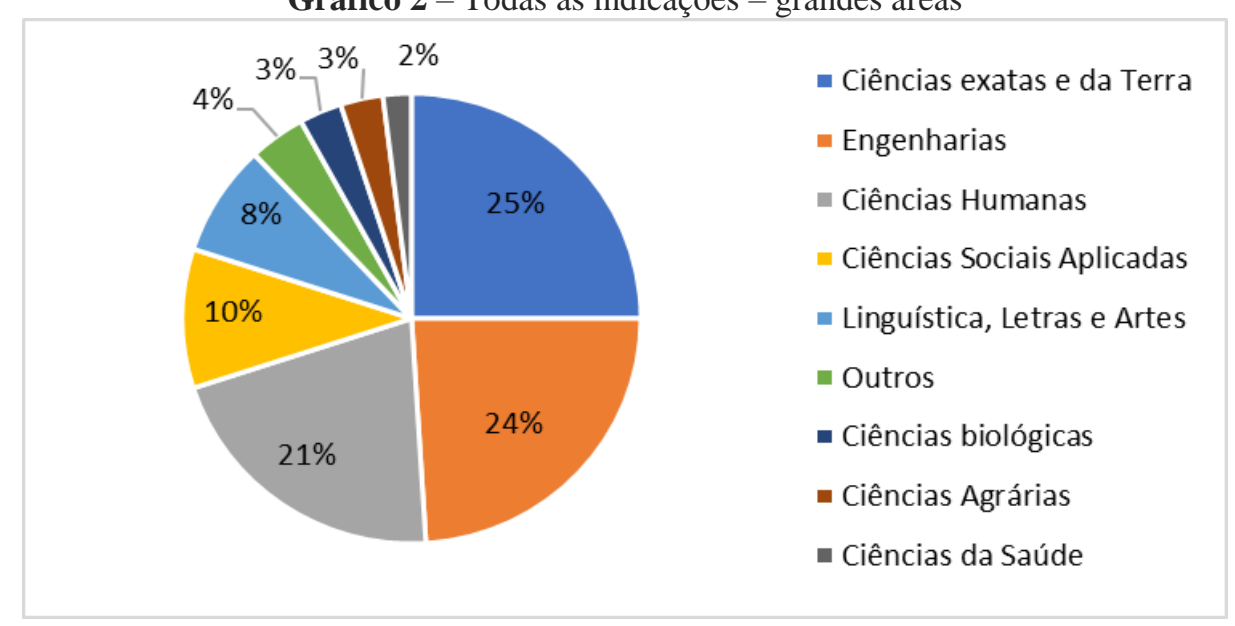

Fonte: Dados da pesquisa. 
Da mesma forma que a primeira indicação representa a descrição sumária do foco disciplinar mais essencial da atuação dos docentes (o que, naturalmente, engloba sua produção científica), o conjunto de todas as indicações é, de certa forma, um indicativo da multidisciplinaridade, pois mostra como os conteúdos disciplinares se conectam entre si por meio de cada docente. $\mathrm{Na}$ verdade, sob a ótica do docente, o preenchimento de várias áreas de atuação é uma declaração de multidisciplinaridade individual, uma vez que mostra a capacidade do docente-pesquisador de atuar em diferentes núcleos disciplinares. Essa conclusão é corroborada por Lança, Amaral e Gracioso (2018), que consideram as declarações das áreas de atuação dos pesquisadores como um indicador de sua multidisciplinaridade.

Por outro lado, a existência de um grande número de docentes com essa inclinação multidisciplinar abre, para o Instituto, diversas possibilidades inter- e transdisciplinares, uma vez que esse tipo de pesquisa pressupõe a existência de indivíduos capazes de transitar entre diferentes disciplinas. Assim, o perfil multidisciplinar dos docentes pode ser um primeiro passo em direção às pesquisas em que haja interação entre as disciplinas, característica da pesquisa interdisciplinar, e/ou de pesquisas sem limites entre as disciplinas, isto é, pesquisas transdisciplinares (PIAGET, 1972).

Através das primeiras indicações, foi possível criar uma mandala contendo as áreas, subáreas e especialidades de cada uma das grandes áreas do IFSP (Gráfico 3). O método de representação por mandalas já foi utilizado por Pinheiro (2018) para demonstrar a interdisciplinaridade da Ciência da Informação. Os indicadores foram produzidos utilizando-se apenas a primeira indicação dos 1997 docentes que preencheram o campo "áreas de atuação" no CVLattes.

Tendo em vista o significado atribuído à primeira indicação pelo próprio CVLattes, a mandala a seguir representa, em tese, as áreas, subáreas e especialidades do IFSP nas quais a pesquisa figura como prioritária para os seus docentes. Do mesmo modo, tais áreas, subáreas e especialidades a priori podem representar a primeira opção de colaboração entre os docentes, vista sua importância para eles. Tal premissa é apoiada por Galdino, Garcia e Amaral 
(2019), que acreditam que apesar da possibilidade de haver colaboração entre os pesquisadores em quaisquer áreas de atuação por eles indicadas, ou até mesmo em uma área sem indicação no CVLattes, há maiores chances de interesse na colaboração na primeira indicação da área de atuação.

Gráfico 3 - Mandala das grandes áreas, áreas, subáreas e especialidades

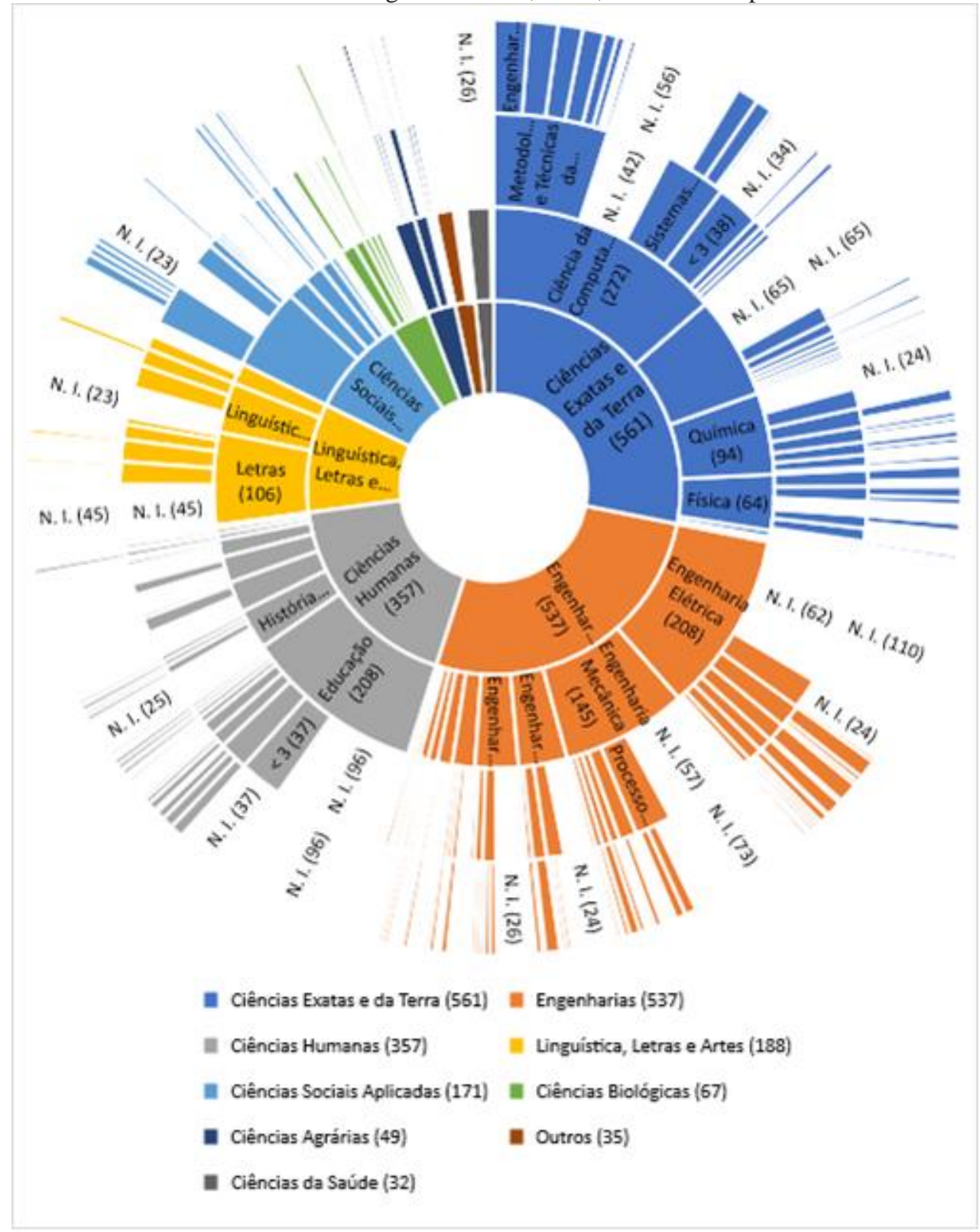

Fonte: Dados da pesquisa.

Dada a propriedade intrínseca da mandala de expressar o tamanho das suas classes e subclasses em função da área no gráfico que elas ocupam, análises 
dessa mandala por simples inspeção visual poderão revelar instantaneamente quais áreas, subáreas e especialidades mais concentram docentes. Essa característica também favorece a escolha de possíveis locus de colaboração, uma vez que quanto maior o número de docentes, maiores as chances de colaboração.

Outra forma de interpretar a mandala é através da análise das suas regiões vazias. Da mesma forma que as regiões coloridas tendem a informar as áreas, subáreas e especialidades de interesse para os docentes, as regiões vazias representariam as "lacunas" de interesse: áreas, subáreas e especialidades sem prioridade na visão dos docentes-pesquisadores. Decorre disso que a chance de haver colaboração nessas áreas, subáreas e especialidades é mais remota.

Por fim, é importante mencionar que a mandala com a primeira indicação tende a indicar a disciplinaridade prevalente no IFSP. Enlaces inter-e transdisciplinares são desejáveis, mas provavelmente envolverão os saberes disciplinares mais relevantes para o Instituto, os quais são representados justamente por essa mandala.

Para o preenchimento da mandala, consideraram-se as áreas, subáreas e especialidades com mais de três pessoas, de forma a não sobrecarregar visualmente as figuras e a não dificultar sua análise. Assim, níveis com menos de três pessoas não foram nomeados, tendo sido representados pela sigla " $<3$ ". Os níveis não preenchidos foram denominados "N. I." (não informado(a)) e estão coloridos de branco. Os valores entre parêntesis "()" correspondem à quantidade de docentes que selecionaram as áreas, subáreas e/ou especialidades indicadas.

Em termos de representatividade, do total de 2214 docentes ativos, 1997 docentes preencheram a primeira indicação de área de atuação do CVLattes, como já mencionado anteriormente. Esse valor equivale a 90,2\% do total, o que torna a amostra bem representativa. O percentual de docentes que escolheram cada grande área foi, por ordem de ocorrência: Ciências Exatas e da Terra (28,5\%), Engenharias (27,3\%), Ciências Humanas (18,2\%), Linguística, Letras e Artes (9,6\%), Ciências Sociais Aplicadas (8,7\%), Ciências Biológicas (3,4\%), Ciências Agrárias (2,5\%), Outros (1,8\%) e Ciências da Saúde $(1,6 \%)$. 
O gráfico revela que a quase totalidade dos docentes que preencheram a grande área também preencheu a área (segundo círculo da mandala, de dentro para fora). Desse modo, é possível dizer que a representatividade das indicações da área também é considerável. Por outro lado, a grande quantidade de espaços em branco no âmbito das subáreas e especialidades indica baixo nível de preenchimento, resultando assim numa amostra de respondentes menor e de menor representatividade do perfil do IFSP. Apenas 58,4\% dos docentes indicaram sua subárea e somente $27,8 \%$ indicaram a especialidade.

Os mapas de correlação cruzada foram utilizados para as próximas análises por tornarem visíveis as variáveis, suas correlações (caso existam) e o grau de intensidade dessas correlações (CANONGIA et al., 2004). Tais mapas já foram usados nos estudos bibliométricos para, entre outros fins, analisar a formação de clusters e as conexões em seu interior (NININ et al., 2015), para analisar a formação de clusters através de referências citadas por um grupo de pesquisadores (MOTTA et al., 2011) e para visualizar redes de colaboração entre instituições (CANONGIA et al., 2004).

No indicador retratado na Figura 1 (Mapa de correlação cruzada: campi $\mathrm{x}$ áreas), as esferas representam cada campus e o diâmetro se refere à quantidade de áreas selecionadas pelos docentes. As linhas entre as esferas representam as relações entre os campi, e a espessura dessas linhas representa a intensidade de tais relações. Já a ausência de linhas entre as esferas demonstra que não há relações entre os campi. Com relação à distância entre as esferas, os campi mais correlacionados são aproximados na figura, enquanto os não correlacionados são distanciados.

Através desse indicador é possível identificar as relações entre os campi por meio das linhas e dos clusters. Observa-se a formação de um único cluster com 19 campi: BRI-Birigui, SBV-São João da Boa Vista, GRU-Guarulhos, CTD-Catanduva, BTV-Boituva, SLT-Salto, SRT-Sertãozinho, PEP-Presidente Epitácio, ARQ-Araraquara, HTO-Hortolândia, VTP-Votuporanga, CMPCampinas, PRC-Piracicaba, BRA-Bragança Paulista, SPO-São Paulo, ITPItapetininga, CBT-Cubatão, SZN-Suzano e CJO-Campos do Jordão. 


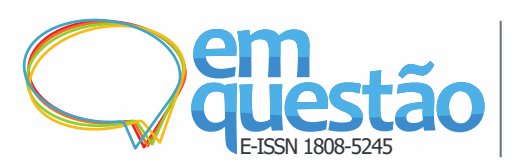

Áreas de atuação de pesquisadores como input para caracterizar a atuação institucional

Rosangela Galdino, Roniberto Morato do Amaral

Figura 1 - Mapa de correlação cruzada: campi x áreas
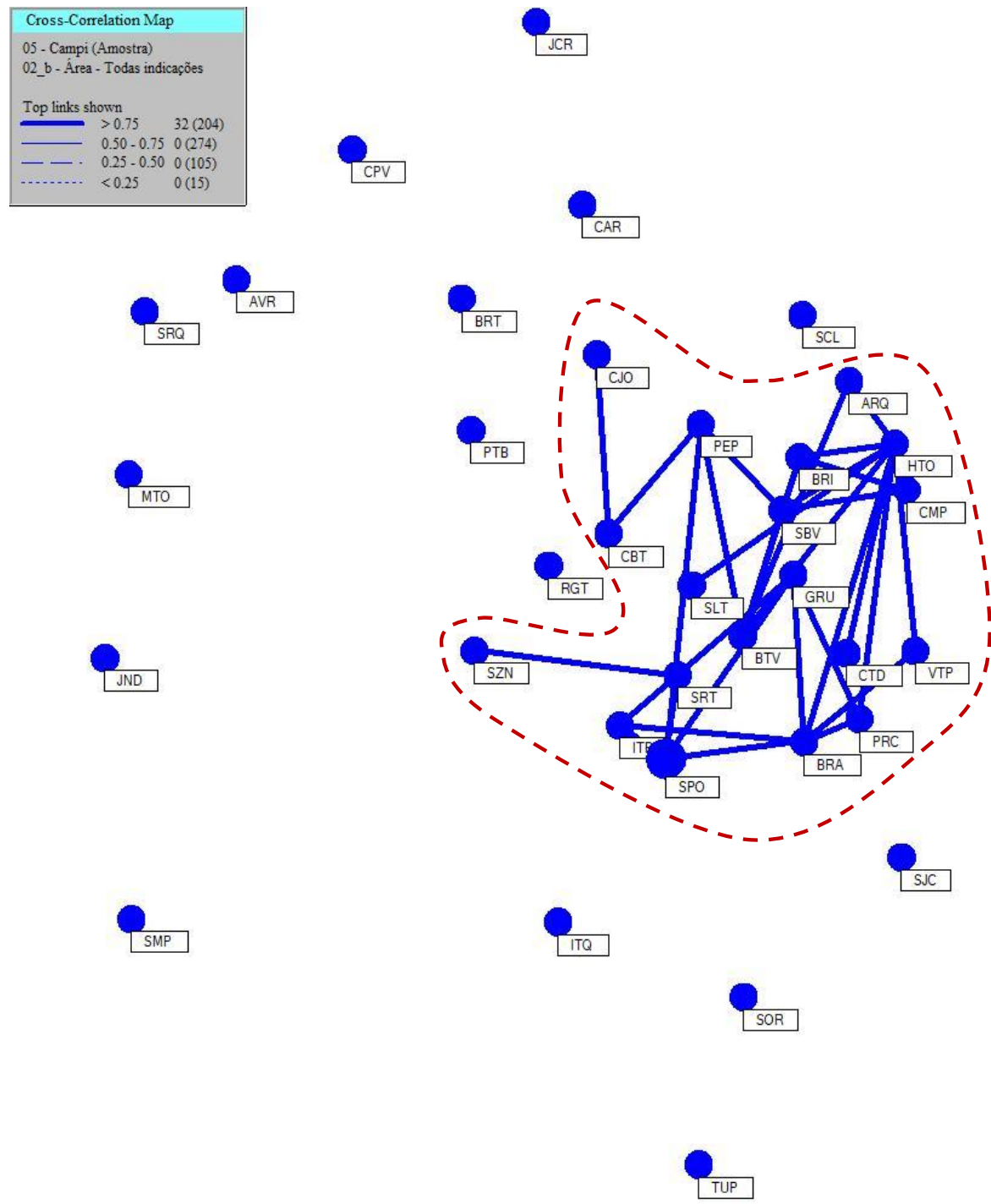

Fonte: Dados da pesquisa.

Através da espessura das linhas, é possível observar que as relações têm a mesma intensidade. Ao mesmo tempo, o diâmetro da esfera que representa São Paulo é maior que o das demais, pois a quantidade de áreas indicadas do campus de São Paulo foi maior (o campus tem maior quantidade de docentes, logo a quantidade de áreas indicadas por eles é maior).

Os campi mais distantes ou isolados não têm nenhuma relação com os outros campi (SCL-São Carlos, RGT-Registro, PTB-Pirituba, BRT-Barretos, 
CAR-Caraguatatuba, SJC-São José dos Campos, ITQ-Itaquaquecetuba, SORSorocaba, TUP-Tupã, JCR-Jacareí, CPV-Capivari, AVR-Avaré, SRQ-São Roque, MTO-Matão, JND-Jundiaí, SMP-São Miguel Paulista e IST-Ilha Solteira).

Visto que há menor pulverização dos docentes nas áreas, devido ao número relativamente pequeno de áreas, várias delas têm representantes nos diversos campi. Desse modo, a correlação entre os campi por meio das áreas apresenta várias conexões.

Na Figura 2 (Mapa de correlação cruzada: campi x subáreas), o indicador retratado possui a mesma moldura analítica da Figura 1. Assim, as esferas representam cada campus e o tamanho delas se refere à quantidade de subáreas selecionadas pelos docentes. Já as linhas entre as esferas representam as relações entre os campi, a sua espessura representa a intensidade dessas relações e a ausência de linhas representa a falta de relações. Além disso, os campi mais correlacionados são aproximados e os não correlacionados são afastados na figura.

É possível identificar, na Figura 2, as relações entre os campi por meio das linhas e dos clusters. Desse modo, nota-se a formação de um único cluster com 16 campi (ARQ-Araraquara, HTO-Hortolândia, BRI-Birigui, VTPVotuporanga, SPO-São Paulo, GRU-Guarulhos, SRT-Sertãozinho, ITPItapetininga, SBV-São João da Boa Vista, CBT-Cubatão, CMP-Campinas, BRA-Bragança Paulista, PRC-Piracicaba, SJC-São José dos Campos, SLT-Salto e SCL-São Carlos), interligados pelas subáreas selecionadas pelos docentes.

Pode-se observar que a ligação entre o campus de Araraquara e o de Hortolândia é mais intensa, representada pela linha mais espessa que une as respectivas esferas. As ligações entre os demais campi têm a mesma intensidade (as linhas possuem a mesma espessura). Além disso, os campi que não têm relação com os outros estão mais distantes ou isolados (CTD-Catanduva, CARCaraguatatuba, BTV-Boituva, PEP-Presidente Epitácio, ITQ-Itaquaquecetuba, SOR-Sorocaba, TUP-Tupã, JCR-Jacareí, CPV-Capivari, MTO-Matão, AVRAvaré, SRQ-São Roque, RGT-Registro, PTB-Pirituba, BRT-Barretos, SZN- 
Suzano, CJO-Campos do Jordão, JND-Jundiaí, SMP-São Miguel Paulista e ISTIlha Solteira).
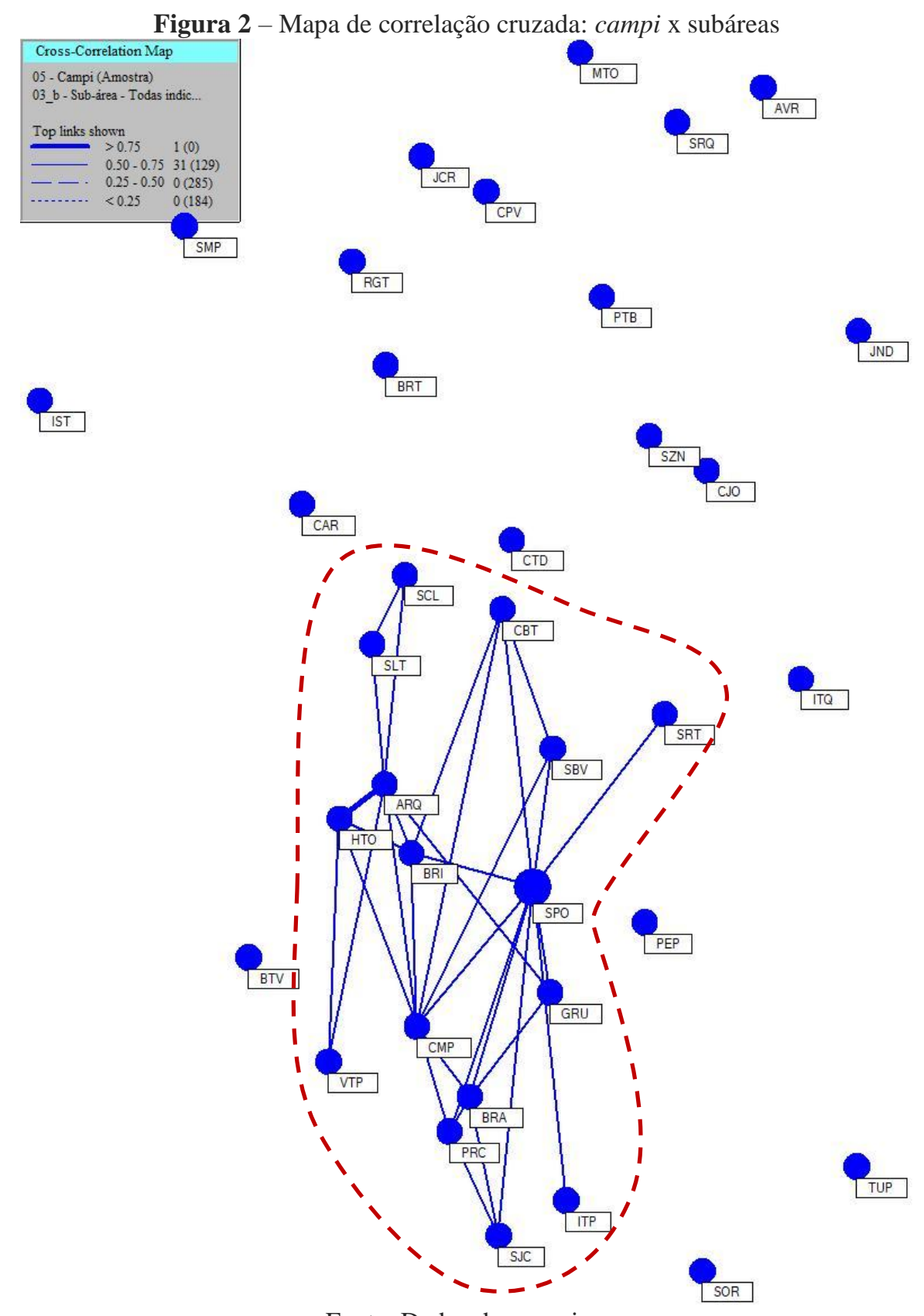

Fonte: Dados da pesquisa.

Nota-se, ao se compararem as figuras 1 e 2, uma queda na correlação, expressa tanto pela redução de campi no cluster quanto pela diminuição da espessura das linhas que conectam as respectivas esferas. Isso ocorre porque a pulverização dos docentes é maior nas subáreas que nas áreas, devido ao número 
das subáreas serem maiores que o das áreas e por haver menor preenchimento das subáreas. Desse modo, quanto mais específica se torna a área de atuação, menor a sua correlação com os campi.

\section{Considerações finais}

Através da pesquisa foi possível alcançar o objetivo do estudo, identificando o perfil de atuação de uma ICT. Para tanto, utilizamos indicadores bibliométricos gerados por meio das informações do campo "área de atuação" dos CVLattes dos docentes-pesquisadores da unidade-caso selecionada, o IFSP.

Dado que esta pesquisa pertence à categoria dos estudos bibliométricos, que caracterizam as instituições e que geram inputs para a melhoria da sua produção científica por meio de análises dos currículos dos seus pesquisadores, é preciso ressaltar a importância do preenchimento adequado do CVLattes. Ao longo de toda a discussão, essa necessidade se fez evidente, revelando que tanto a qualidade das análises quanto a abrangência das conclusões dependiam do correto e completo preenchimento dos currículos dos pesquisadores. Tendo em vista que o CVLattes vem sendo utilizado cada vez mais como fonte de informação pelas instituições de ensino, por pesquisadores e por agências de fomento e pesquisa, é necessário que sejam planejadas e implementadas iniciativas que sensibilizem os pesquisadores sobre a importância do correto preenchimento. Tais iniciativas devem estimular a completude dos dados dos docentes sobre a sua atuação em ensino, pesquisa e extensão na Plataforma Lattes e ainda conscientizá-los sobre o impacto desses dados nas análises e na compreensão da atuação institucional.

No que diz respeito à análise da área de atuação nos CVLattes, a mandala de primeira indicação revela a disciplinaridade dos docentes do IFSP, pois considerou-se apenas as primeiras indicações dos docentes em seus CVLattes de grandes áreas, áreas, subáreas e especialidades do conhecimento, definidas pelo $\mathrm{CNPq}$ na Plataforma Lattes. Essas indicações, entretanto, representam também o caráter multidisciplinar do IFSP, uma vez que a instituição é constituída de todas as áreas de atuação dos docentes. Por fim, analisou-se a correlação entre os campi e o campo "áreas de atuação" (figuras 1 
e 2). Assim, verificou-se que quanto maior a especificidade da área de atuação, maior a pulverização dos campi e menor a correlação entre eles, demonstrada tanto pela redução de campi no cluster quanto pela diminuição da espessura das linhas que conectam as esferas que representam os campi. Isso decorre do fato de que vários docentes não especificam completamente suas áreas de atuação, optando por registrar apenas a grande área e a área.

Além disso, através da comparação das figuras 1 e 2, é possível ver quais campi se repetem em ambos os clusters e apontar os campi dos quais mais se poderia esperar uma colaboração no IFSP, a saber: Araraquara, Hortolândia, Birigui, Votuporanga, São Paulo, Guarulhos, Sertãozinho, Itapetininga, São João da Boa Vista, Cubatão, Campinas, Bragança Paulista, Piracicaba, São José dos Campos, Salto e São Carlos. Através dessas informações o Instituto poderia aproveitar as possibilidades de colaboração entre os campi e fazer intervenções que estimulassem essa colaboração, tais como grupos de pesquisa multicampi e realização de eventos por área.

É importante ressaltar que, apesar de dois ou mais pesquisadores pertencerem a uma mesma área de atuação, isso não significa que haverá colaboração entre eles, haja vista que há mais fatores que devem ser considerados. Nesse sentido, para futuros trabalhos seria interessante o estudo de outros fatores além das áreas de atuação fornecidas no CVLattes dos pesquisadores, tais como os temas de interesse, a localização geográfica, as palavras-chaves de pesquisas já realizadas e outros fatores que possam influenciar na colaboração científica. Além disso, a pesquisa também poderá ser aplicada a outras ICTs (até mesmo outros IFs) que desejem conhecer o próprio perfil de atuação e o de seus pesquisadores com o intuito de ampliar o sucesso do projeto institucional, a produtividade e a qualidade das pesquisas.

Conclui-se que os resultados alcançados podem contribuir para os estudos bibliométricos que utilizam a Plataforma Lattes como fonte de coleta de dados para gerar inputs informacionais sobre a atuação institucional e para buscar formas de melhorias na produção científica ao sistematizar, através da análise das áreas de atuação dos pesquisadores, a identificação e visualização do 
perfil de atuação de uma ICT, bem como a identificação de áreas cujo interesse para colaboração entre docentes é potencialmente maior.

\section{Referências}

BENTIN, P. O ensino de graduação nos Institutos Federais. In: ANJOS, M. B. dos; RÔÇAS, G. (org.). As políticas públicas e o papel social dos Institutos Federais de Educação, Ciência e Tecnologia. Natal: IFRN, 2017. p. 13-35.

BORDONS, M.; ZULUETA M. A. Evaluación de la actividad científica através de indicadores bibliométricos. Revista Española de Cardiología, Madrid, v. 52, n. 10, p. 790-800, oct. 1999.

BRASIL. Decreto $\mathbf{n}^{\mathbf{0}} \mathbf{7 . 5 6 6}$, de 23 de setembro de 1909. Crêa nas capitaes dos Estados da Republica Escolas de Aprendizes Artífices, para o ensino profissional primario e gratuito. Rio de Janeiro: Presidência da República, 1909.

BRASIL. Lei $\mathbf{n}^{\mathbf{0}}$ 11.892, de 29 de dezembro de 2008. Institui a Rede Federal de Educação Profissional, Científica e Tecnológica, cria os Institutos Federais de Educação, Ciência e Tecnologia, e dá outras providências. Brasília, DF: Presidência da República, [2018].

BRASIL. Ministério da Educação. Instituto Federal de Educação, Ciência e Tecnologia: um novo modelo em educação profissional e tecnológica. Concepção e Diretrizes. [Brasília]: [Presidência da República], 2010.

BRITO, A. G. C. de; QUONIAM, L.; MENA-CHALCO, J. P. Exploração da Plataforma Lattes por assunto: proposta de metodologia. Transinformação, Campinas, v. 28, n. 1, p. 77-86, jan./abr. 2016.

CANONGIA, C. et al. Mapeamento de inteligência competitiva (IC) e de gestão do conhecimento (GC) no setor saúde. Encontros Bibli: revista eletrônica de biblioteconomia e ciência da informação, Florianópolis, p. 78-95, jan. 2004.

DOMINGOS SOBRINHO, M. Universidade Tecnológica ou Instituto Federal de Educação, Ciência e Tecnologia? [Brasília]: Ministério da Educação, [2007].

FARTES, V. L. B. A cultura profissional dos grupos de pesquisa nos institutos federais: uma comunidade de práticas? Cadernos de Pesquisa, São Paulo, v. 44, n. 154, p. 850-874, 2014.

FRANCO, N. M. G.; FARIA, L. I. L. de. Colaboração científica intraorganizacional: análise de redes por coocorrência de palavras-chave. Em Questão, Porto Alegre, v. 25, n. 1, p. 87-110, jan./abr. 2019. Disponível em: https://seer.ufrgs.br/EmQuestao/article/view/79906/0. Acesso em: 20 nov. 2019. 
GALDINO, R.; GARCIA, L. G.; AMARAL, R. M. Áreas de atuação como input para potenciais parcerias entre os docentes do Instituto Federal de São Paulo (IFSP). In: ENCONTRO NACIONAL DE PESQUISA EM CIÊNCIA DA INFORMAÇÃO, 20, 2019. Florianópolis. Anais [...]. Florianópolis: ENANCIB, 2019.

GIL, A. C. Como elaborar projetos de pesquisa. 5. ed. São Paulo: Atlas, 2010.

HADDAD, E. A.; MENA-CHALCO, J. P.; SIDONE, O. J. G. Produção científica e redes de colaboração dos docentes vinculados aos programas de pósgraduação em Economia no Brasil. Estudos Econômicos, São Paulo, v. 47, n. 4, p. 617-679, out./dez. 2017.

JANTSCH, E. Towards interdisciplinarity and transdisciplinarity in education andinnovation. In: APOSTEL, Léo et al. Interdisciplinarity: problems of teaching and researchin universities. Paris: Centre for Educational Research and Innovation, 1972. p. 97-121.

JAPIASSU, H. Interdisciplinaridade e a patologia do saber. Rio de Janeiro: Imago, 1976.

KATZ, J. S.; MARTIN, B. R. What is research collaboration? Research Policy, Amsterdam, v. 26, n. 1, p. 1-18, 1997.

LANÇA, T. A.; AMARAL, R. M.; GRACIOSO, L. S. Multi e interdisciplinaridade nos programas de pós-graduação em ciência da informação brasileiros. Perspectivas em Ciência da Informação, Belo Horizonte, v. 23, n. 4, out./dez. 2018.

LANE, J. Let's make science metrics more scientific. Nature, London, v. 464, n. 7288, p. 488-489, mar. 2010.

MAIA, M. de F. S.; CAREGNATO, S. E. Coautoria como indicador de redes de colaboração científica. Perspectivas em Ciência da Informação, Belo Horizonte, v. 13, n. 2, p. 18-31, maio/ago. 2008.

MATIAS, M. S. O. Base referencial para o povoamento de repositórios institucionais: coleta automatizada de metadados da Plataforma Lattes. 2015. Dissertação (Mestrado em Gestão de Organizações e Sistemas Públicos) Universidade Federal de São Carlos, São Carlos, 2015.

MENA-CHALCO, J. P; DIGIAMPIETRI, L. A.; CESAR JUNIOR, R. M. Caracterizando as redes de coautoria de currículos Lattes. In: BRAZILIAN WORKSHOP ON SOCIAL NETWORK ANALYSIS AND MINING, 1., 2012, Curitiba. Anais eletrônicos [...] Curitiba: BrasNAM, 2012. 
MOTTA, G. et al. O perfil da pesquisa acadêmica sobre jogos de empresas entre 2001 e 2010. In: ENCONTRO DA ANPAD, 35., 2011, Rio de Janeiro. Anais eletrônicos [...]. Rio de Janeiro: ENANPAD, 2011. p. 1-16.

NININ, D. M. et al. Indicadores de circulação do acervo na Biblioteca Comunitária da Universidade Federal de São Carlos. Transinformação, Campinas, v. 27, n. 1, p. 59-71, abr. 2015.

PIAGET, J. The epistemology of interdisciplinary relationship. In: APOSTEL, L. et al. Interdisciplinarity: problems of teaching and research in universities. Paris: Centre for Educational Research and Innovation, 1972. p. 127-140.

PINHEIRO, L. V. R. Mutações na ciência da informação e reflexos nas mandalas interdisciplinares. Informação \& Sociedade: Estudos, João Pessoa, v. 28, n. 3, p. 115-134, set./dez. 2018.

PORTER, A.; PALOP, F. Mineração de textos para decisões de gestão de pesquisa e tecnologia - tech mining com a ajuda de software VantagePoint. In: ENCONTRO BRASILEIRO DE BIBLIOMETRIA E CIENTOMETRIA, 3., Gramado. Anais eletrônicos [...]. Gramado: UFRGS, 2012. p. 1-26.

ROSTAING, H. La bibliométrie et ses techniques. Tolouse: Sciences de la Société, 1996.

SALA, O. A questão da ciência no Brasil. Estudos Avançados, São Paulo, v. 5, n. 12, p. 153-160, mai./ago. 1991.

SIDONE, O. J. G.; HADDAD, E. A.; MENA-CHALCO, J. P. A ciência nas regiões brasileiras: evolução da produção e das redes de colaboração científica. Transinformação, Campinas, v. 28, n. 1, p. 15-32, jan./abr. 2016.

SOARES, P. C. Contradições na pesquisa e pós-graduação no Brasil. Estudos Avançados, São Paulo, v. 32, n. 92, p. 289-313, 2018.

TORRES, M. F. N. Contribuição à identificação da competência científica de um Instituto Público de Pesquisa por meio de indicadores bibliométricos. 2018. Tese (Doutorado em Ciência, Tecnologia e Sociedade) Universidade Federal de São Carlos, São Carlos, 2018.

VANTAGEPOINT. VantagePoint user's guide. Norcross, Georgia: Search Technology, 2015.

VANZ, S. A. de S.; STUMPF, I. R. C. Colaboração científica: revisão teóricoconceitual. Perspectivas em Ciência da Informação, Belo Horizonte, v. 15, n. 2, p. 42- 55, maio/ago. 2010. 


\title{
Research areas as input to characterize institutional activities
}

\begin{abstract}
Bibliometric studies are used in the evaluation of scientific activity. They may gather data from databases such as Web of Science and Scopus, and, in Brazil, the Lattes Platform. This case study aimed to help describe the performance of science, technology and innovation institutions through bibliometric procedures, and, specifically, to outline the performance of the Federal Institute of Education, Science and Technology of São Paulo (IFSP) using data about the areas of activity of its teachers-researchers available on the Lattes Plataform. The analyzed sample comprised 1997 Lattes curricula vitae of -teachers-researchers active in the period from 2010 to 2018. The institute's academic output was found to be multidisciplinary, and some activity between campuses was found to be clustered. The results of this study could contribute to the bibliometric study of science, technology and innovation institutions by profiling one such institution, through analysis of the research interests of its researchers, and pointing areas in which its researchers would be most likely to collaborate.
\end{abstract}

Keywords: Area of expertise. Lattes curriculum. Instituto Federal de Educação, Ciência e Tecnologia de São Paulo.

Recebido: 09/12/2019

Aceito: 08/06/2020

\section{Como citar}

GALDINO, Rosangela; AMARAL, Roniberto Morato do. Áreas de atuação de pesquisadores como input para caracterizar a atuação institucional. Em

Questão, Porto Alegre, v.27, n. 1, p. 286-307, 2021. Doi:

https://doi.org/10.19132/1808-5245271.286-307 